\title{
Impact of Radio Irregularity on Wireless Sensor Networks
}

\author{
Gang Zhou, Tian He, Sudha Krishnamurthy, John A. Stankovic \\ Department of Computer Science \\ University of Virginia, Charlottesville, VA 22903 \\ \{gz5d, tianhe, skrish, stankovic\}@cs.virginia.edu
}

\begin{abstract}
In this paper, we investigate the impact of radio irregularity on the communication performance in wireless sensor networks. Radio irregularity is a common phenomenon which arises from multiple factors, such as variance in RF sending power and different path losses depending on the direction of propagation. From our experiments, we discover that the variance in received signal strength is largely random; however, it exhibits a continuous change with incremental changes in direction. With empirical data obtained from the MICA2 platform, we establish a radio model for simulation, called the Radio Irregularity Model (RIM). This model is the first to bridge the discrepancy between spherical radio models used by simulators and the physical reality of radio signals. With this model, we are able to analyze the impact of radio irregularity on some of the well-known MAC and routing protocols. Our results show that radio irregularity has a significant impact on routing protocols, but a relatively small impact on MAC protocols. Finally, we propose six solutions to deal with radio irregularity. We evaluate two of them in detail. The results obtained from both the simulation and a running testbed demonstrate that our solutions greatly improve communication performance in the presence of radio irregularity.
\end{abstract}

\section{Categories and Subject Descriptors}

C.2.1 [Computer Communication Network]: Network Architecture and Design; I.6 [Computing Methodologies]: Simulation and Modeling

\section{General Terms}

Design, algorithms, measurement, performance, experimentation

\section{Keywords}

Sensor networks, wireless communication, radio irregularity, sending power, path loss, link asymmetry, packet loss

Permission to make digital or hard copies of all or part of this work for personal or classroom use is granted without fee provided that copies are not made or distributed for profit or commercial advantage and that copies bear this notice and the full citation on the first page. To copy otherwise, or republish, to post on servers or to redistribute to lists, requires prior specific permission and/or a fee.

MobiSYS'04, June 6-9, 2004, Boston, Massachusetts, USA.

Copyright 2004 ACM 1-58113-000-0/00/0004_..\$5.00.

\section{INTRODUCTION}

Radio irregularity is a common and non-negligible phenomenon in wireless sensor networks. It results in irregularity in radio range and variations in packet loss in different directions, and is considered as an essential reason for asymmetric links as viewed by upper layers in the protocol stack. Several empirical studies $[4][9][23][28]$ on the Berkeley mote platform have shown that the radio range varies significantly in different directions and the percentage of asymmetric links in a system varies depending on the average distance between nodes.

The impact of radio irregularity on protocol performance can be investigated through a running system. However, few researchers have actually pursued this direction, because of two reasons: First, the complexity and cost of performance evaluations on a running system escalate, when sensor networks scale up to thousands or more nodes. Second, repeatable results of radio performance are extremely hard to obtain from uncontrolled environments, hence leading to difficulties in system tuning and performance evaluation. As a result, simulation techniques are used as an efficient alterative to evaluate protocol performance. Unfortunately, most existing simulations don't take radio irregularity, a common phenomenon in wireless communication, into account. The spherical radio patterns assumed by simulators such as [27] may not approximate real radio properties well enough and hence may lead to an inaccurate estimation of application performance.

Several researchers [4][9][23][28] have already shown extensive evidence of radio irregularity in wireless communication. Their main focus is to observe and quantify such phenomena. This paper is distinguished from the previous ones for the initiative in bridging the gap between spherical radio models used by simulators and the physical reality of radio signals. We first verify the presence of radio irregularity using empirical data obtained from the MICA2 platform. The results demonstrate that the radio pattern is largely random; however, it exhibits a continuous change with incremental changes in direction. Based on experimental data, a radio model for simulations, called the Radio Irregularity Model (RIM), is formulated. RIM takes into account both the non-isotropic ${ }^{1}$ properties of the propagation media and the heterogeneous properties of devices.

\footnotetext{
${ }^{1}$ Exhibiting properties with different values when measured along axes in different directions.
} 
With the help of the RIM model, we explore the impact of radio irregularity on MAC and routing performance. Among the protocols we evaluate, we find that radio irregularity has a significant impact on the routing protocols, but a relatively small impact on the MAC protocols. We also find that location-based routing protocols, such as Geographic Forwarding (GF) [17] perform worse in the presence of radio irregularity than ondemand protocols, such as AODV [21] and DSR [14]. We propose several potential solutions to deal with radio irregularity in wireless sensor networks. We evaluate two of them through a simulation and a running system, respectively. Our results illustrate that our solutions do succeed in alleviating the performance penalties due to radio irregularity.

The rest of this paper is organized as follows: we briefly analyze the causes and impact of radio irregularity in Section 2. In Section 3, we describe experimental data collected from the MICA2 platform and make some general conclusions about radio irregularity. Based on these conclusions, we propose the RIM radio model in Section 4. We then use the RIM model in simulations to analyze the impact of radio irregularity on MAC and network layer protocols in Section 5 and Section 6, respectively. Solutions to deal with radio irregularity are proposed and evaluated in Section 7. Finally, we conclude the paper in Section 8 .

\section{ANALYSIS OF RADIO IRREGULARITY}

In this section, we first identify the causes of radio irregularity, and then briefly discuss the impact of irregularity on the different protocol layers.

\subsection{Causes of Radio Irregularity}

Radio irregularity is caused by two categories of factors: devices and the propagation media. Device properties include the antenna type (directional or omni-directional), the sending power, antenna gains (at both the transmitter and receiver), receiver sensitivity, receiver threshold and the Signal-Noise Ratio (SNR). Media properties include the media type, the background noise and some other environmental factors, such as the temperature and obstacles within the propagation media.

In general, the radio irregularity is caused by the non-isotropic properties of the propagation media and the heterogeneous properties of devices. Among all these factors, we focus on the non-isotropic path losses and the differences in signal sending power, which are commonly regarded as the key causes of radio irregularity.

- Non-isotropic Path Losses: The variance in the signal path loss is one of the major causes of radio irregularity. When a signal propagates within a medium, it may be reflected, diffracted, and scattered [22]. Reflection occurs when an electromagnetic signal encounters an object, such as a building, that is larger than the signal's wavelength. Diffraction occurs when the signal encounters an irregular surface, such as a stone with sharp edges. Scattering occurs when the medium through which the electromagnetic wave propagates contains a large number of objects smaller than the signal wavelength. The medium is normally different in different directions. Consequently, radio propagation exhibits non-isotropic patterns in most environments.
Another source of non-isotropic path loss lies in hardware calibration. A node may not have the same antenna gain along all propagation directions, possibly due to hardware manufacturing. Hence, the non-isotropic antenna gain of each node also contributes to the non-isotropic path loss.

- Heterogeneous Sending Powers: Sensor devices may transmit RF signal at different sending powers, even though they are the same kind of devices. This difference may arise from some random factors during the manufacture of sensor devices. In addition, after the sensor devices are deployed, the batteries of different sensor devices deplete at different rates, due to different workloads and different environments in which they are deployed. Heterogeneous sending powers result in variable communication ranges, and cause nonisotropic connectivity.

\subsection{Impact of Radio Irregularity}

Radio irregularity is a non-negligible phenomenon in wireless communication. It's an essential reason for asymmetric radio interference and asymmetric links in upper layers. It can directly or indirectly affect many aspects of upper layer performance.

Asymmetric radio interference between neighboring nodes affects the correctness of MAC layer functions. For example, in the presence of radio irregularity, a node might not be able to successfully reserve the wireless channel through RTS and CTS handshaking, because those neighboring nodes of the receiver, which can not hear the CTS control packet, might disrupt the receiving node. This impacts the delivery ratios of data frames at the MAC layer.

Radio irregularity can also affect the performance and even correctness of networking protocols such as [12] [14] [16] [17] [21]. For example, link asymmetry is one of the ways in which radio irregularity manifests itself at the higher layer. Link asymmetry has an adverse impact on protocols that use pathreversal techniques to establish an end-to-end connection.

Actually, the impact of radio irregularity is not only confined to the MAC and routing layers, radio irregularity also influences other protocols, such as the localization, sensing converge and topology control protocols.

Localization protocols such as DV-Hop [20] and Centroid [2] assume a spherical radio range. The study in [10] shows that the performance of such protocols degrades when the radio range becomes irregular. The sensing coverage scheme in [26] assumes that sensing and communication ranges are spherical. In the presence of radio irregularity, they might not be able to guarantee full coverage and blind points would occur. The topology-control scheme in GAF [25] builds a communication mesh based on the assumption of a spherical range. This might lead to the network partition in the presence of a non-spherical range. We note that some other topology-control protocols, such as ASCENT [3] and Span [5] don't depend on such an assumption, however, performance evaluations of those protocols considering radio irregularity are desired.

Due to space limitation, in the rest of paper, we only focus on the impact of radio irregularity on MAC and routing performance and leave the rest as future work. 


\section{RADIO IRREGULARITY IN REALITY}

We conduct several experiments to study the irregularity of the radio using MICA2 motes, and in this section we discuss some of the experimental results we obtain in an outdoor environment. Our results confirm that radio propagation is largely non-isotropic and exhibits a continuous variation with incremental changes in direction.

\subsection{Experimental Setup}

We use a pair of MICA2 motes for our experiments. One of the motes periodically transmits probing beacons and the other mote samples its ADC port while receiving these beacons. The ADC reads the signal on the analog pin of the Chipcon transceiver [6] and converts it into a 10-bit voltage value. The voltage reading is mapped into the received signal strength in $\mathrm{dBm}$ according to the specification in [6]. All experiments are conducted in an open parking lot near a building.

\subsection{Experimental Results}

In this section, we demonstrate the presence of radio irregularity using three different metrics: 1) the received signal strength, 2) the packet reception ratio and 3 ) the communication range.

1) Non-isotropic Signal Strength: In the first experiment, the receiver is placed 10 feet away from the sender (both on the ground) and the received signal strength is measured in four different geographical directions by sampling 100 beacons received in each direction.

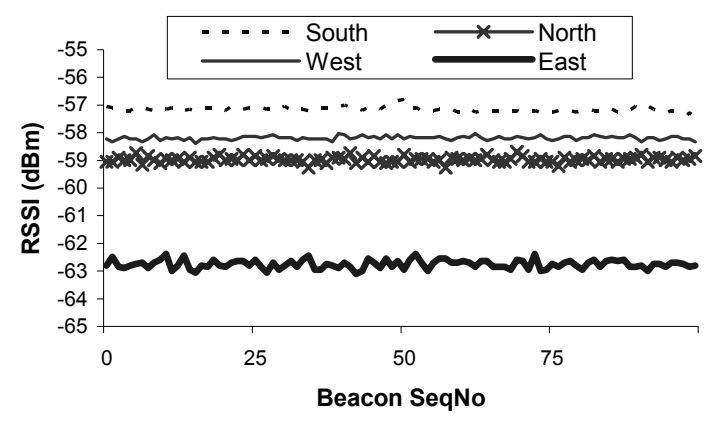

Figure 1: Signal Strength over Time in Four Directions

Figure 1 shows that the received signal strength in each direction is relatively stable over time (The small variance comes from the fading effect [22]). However, the signal strength received in the south is much higher than that received in the east, although nodes have the same distance from the sender. We also measure the variation of signal strength with the changes in the angular direction of the receiver with respect to the sender. Figure 2 shows the variation of the received signal strength as a function of the angular direction with respect to the sender, when the distance between the sender and receiver is 10 feet and 20 feet, respectively. These results show that the received signal strength varies continuously with the direction. In other words, incremental changes in direction result in incremental variation in the received signal strength.
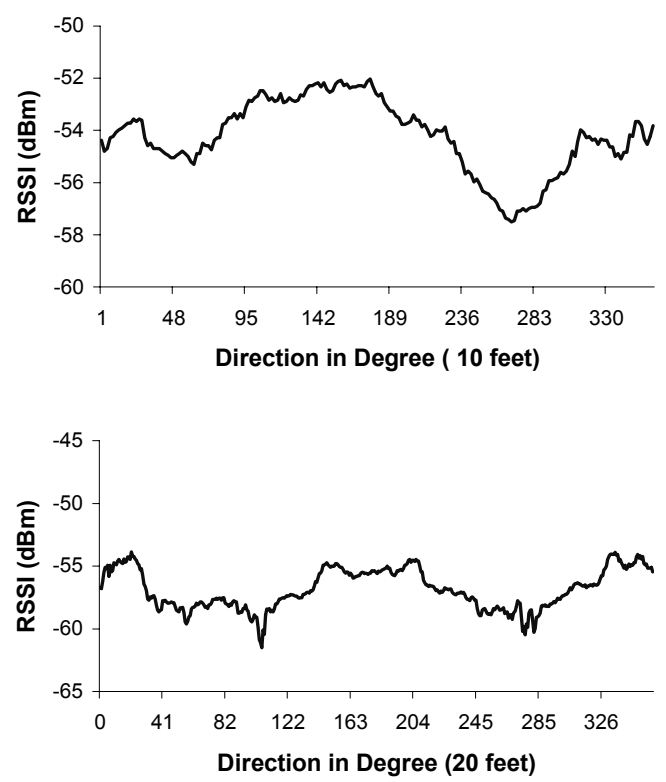

Figure 2: Signal Strength Values in Different Directions

2) Non-isotropic Packet Loss Ratio: Figure 3 shows how the packet reception ratio varies in different directions. When the sender and receiver are placed 10 feet apart, the packet reception ratio is nearly $100 \%$ in all the directions, because the signal is still strong in all the directions. However, when they are placed 20 feet apart, there is a $90 \%$ packet loss in the east direction. This result is consistent with the results shown in Figure 1, which demonstrates that the received signal strength measured in the east is lower than that in the other three directions.

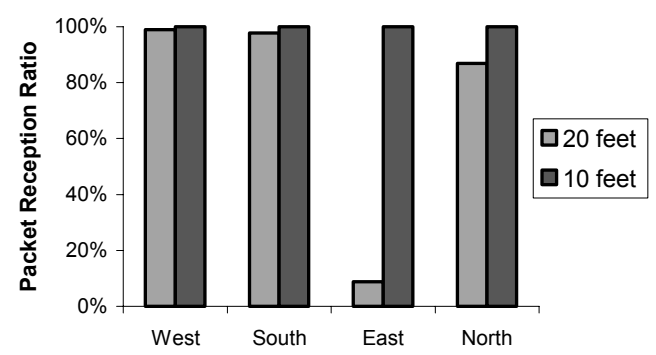

Figure 3: Non-isotropic Packet Reception

3) Non-isotropic Radio Range: Another aspect in which we demonstrate the irregularity is to show that the communication range of a mote is not uniform in all directions. In the experiment, we fix the received signal strength threshold at $-55.5 \mathrm{dBm}$ and -59 $\mathrm{dBm}$, respectively. Then with such thresholds, we measure the communication ranges in different directions. Figure 4 shows the communication range of a mote as the receiver direction varies from degree 0 to degree 359. The range map shown in Figure 4 is another confirmation of radio irregularity in a wireless medium. 


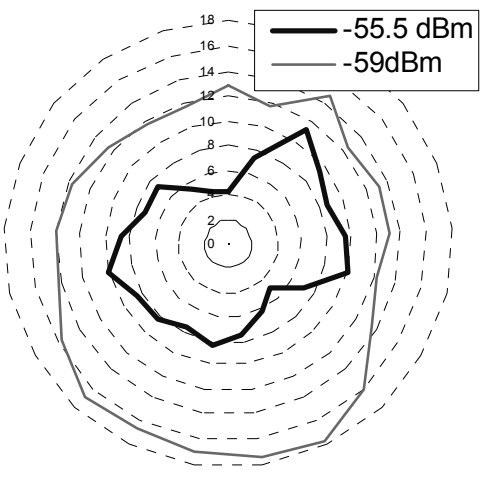

Figure 4: Non-isotropic Range

4) Range Irregularity with Varying Sending Power: We also investigate the received signal strength when the sending power varies due to different battery status and different hardware calibration. In Figure 5 (a), we use the same sender and receiver, placed 10 feet apart. We change the batteries at the sender side each time. The result indicates that different battery status at the same sender can affect the received signal strength. In Figure 5 (b), we use the same batteries but in different senders each time. The same receiver is used, placed 10 feet apart from the sender. The result shows that different senders with the same batteries can also affect the received signal strength.

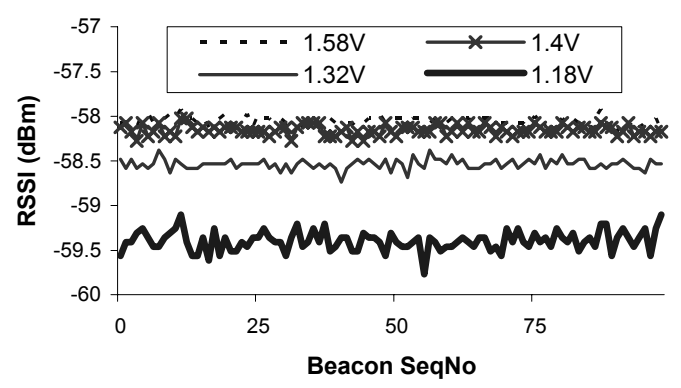

(a) One mote with different battery status

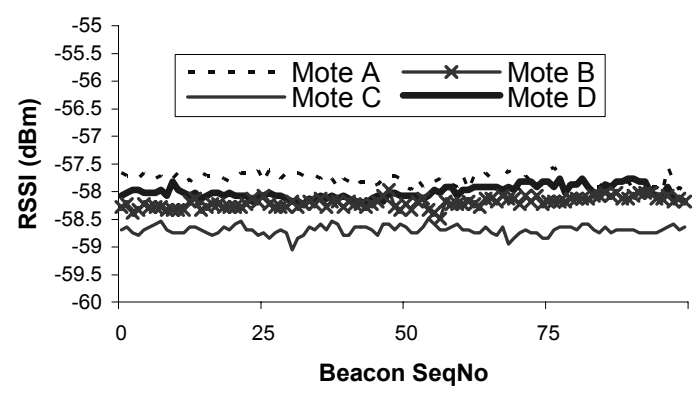

(b) Different motes with the same battery status

Figure 5: Radio Irregularity with Sending Powers

\subsection{Summary of Experimental Results}

From the experimental results, we infer that the radio of sensor devices has the following main properties:

1. Non-isotropic: The radio signal from a transmitter has different path loss in different directions (Figure 1 and Figure 2 ).

2. Continuous variation: The signal path loss varies continuously with incremental changes of the propagation direction from a transmitter (Figure 2 and Figure 4).

3. Heterogeneity: Differences in hardware calibration and battery status lead to different signal sending powers, hence different received signal strengths (Figure 5).

\section{MODEL THE RADIO IRREGULARITY}

As we have shown in our experiments as well as in other research results [4] [9] [23] [28], radio irregularity is a common phenomenon in wireless sensor networks. Therefore, it is essential for wireless simulations to capture such effects. This section describes our effort to model such a phenomenon in simulation environments.

\subsection{Isotropic Radio Models}

In isotropic radio models, the received signal strength is usually represented with the following formula:

Received Signal Strength $=$ Sending Power-Path Loss+Fading (1)

The Sending Power of a node is determined by the battery status and the type of transmitter, amplifier and antenna. Path Loss describes the signal's energy loss as it travels to the receiver. Many models are used to estimate the Path Loss, such as the freespace propagation model, the two-ray model and the Hata model [22]. All these models are isotropic, meaning that the signal attenuates exactly the same in all directions. However, our experience as well as results obtained by others [4] [9] [23] [28] all indicate that the isotropic models don't hold well in practice.

\subsection{Radio Irregularity Model (RIM)}

The RIM model proposed here is an extension to isotropic radio models. It enhances isotropic radio models by approximating three main properties of radio signals: non-isotropic, continuous variation and heterogeneity, as we summarized in section 3.3. These properties are normally ignored by previous isotropic radio models.

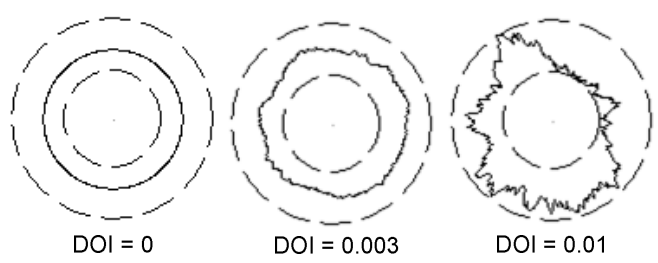

Figure 6: Degree of Irregularity

The RIM model is motivated by a simple DOI (Degree of Irregularity) model briefly mentioned in the localization work [10]. In the DOI model, the DOI is used to denote the irregularity of the radio pattern. It was originally defined as the maximum range variation per unit degree change in the direction of radio propagation. As seen in Figure 6, when the DOI is set to zero, 
there is no range variation, and the communication range is a perfect circle. However, when we increase the DOI value, the communication range becomes more and more irregular.

The DOI model assumes an upper and lower bound on signal propagation (Figure 6). Beyond the upper bound, all nodes are out of communication range; and within the lower bound, every node is guaranteed to be within the communication range. If the distance between a pair of nodes is between these two boundaries, three scenarios are possible: 1) symmetric communication, 2) asymmetric communication, and 3) no communication.

The DOI model is a good start to model signal irregularity. However, it doesn't model interference in real devices well. Since the DOI model is based on an absolute communication range, it assumes that within the inner range, the signal is very strong and can always be received correctly, while beyond the outer range there is no signal at all. This binary pattern is not true in reality. For example, in Figure 7 (a), the DOI model assumes that there is no interference between nodes B and C.

However in reality, there are no such clear boundaries and the communications of nodes do interfere with each other. Different from the DOI model, the RIM model proposed here takes the radio sending energy, the energy loss, the background noise, and the interference among different communication signals into account.

The difference can be further explained with an example. In Figure 7 (b), the RIM model allows node B's signal to propagate beyond its communication range to reach node $\mathrm{C}$, even though it is not strong enough for node $\mathrm{C}$ to receive it as a valid packet. This weak signal from node $\mathrm{B}$ acts as one source of background noise around node $\mathrm{C}$. In this case, node $\mathrm{C}$ may not be able to receive packets from node $A$, if the received signal is not stronger than the product of the Signal-Noise Ratio (SNR) value and background noise level of node $\mathrm{C}$.
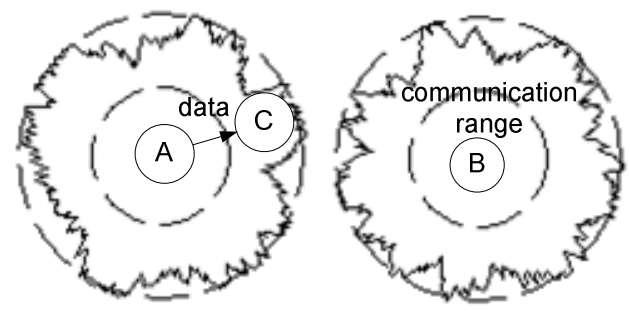

(a) No interference in the DOI model

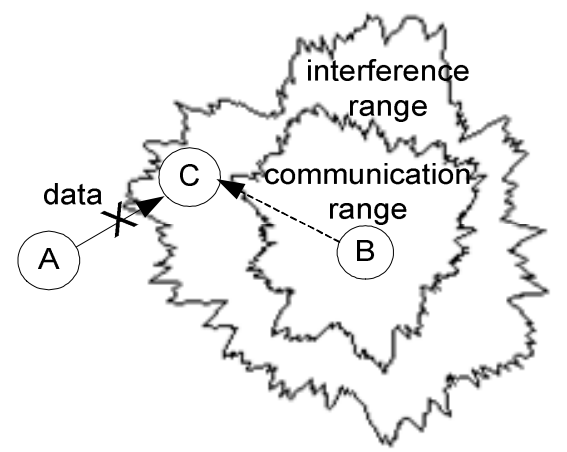

(b) Interference in the RAM energy model

Figure 7: Communication Interference
The DOI model only models an absolute range based on the distance and determines whether one node can hear another node only by comparing the distances between these two nodes with the sender's communication range. With such a binary decision, it can't deal with interference as we mentioned earlier.

To enhance the isotropic radio model and the DOI radio model, we propose the RIM model that combines the energy models and the DOI factor together. We redefine DOI in the RIM model as the maximum received signal strength percentage variation per unit degree change in the direction of radio propagation. We note that RIM is a general radio model which can default to the isotropic model when the DOI value is zero. Also, it can default to the DOI model when there is no interference among nodes.

We note that our RIM model is established based on data from real sensor devices. It is a hybrid approach, which introduces real data (DOI value) into simulations, so that the radio irregularity pattern in reality can be approximated well. DOI values can be calculated according to its definition. We repeat the experiments shown in Figure 2 six times and the results are in Figure 8. It shows that the variances of the received signal strength with incremental changes in direction are small, which validates our conclusion about continuous variation.

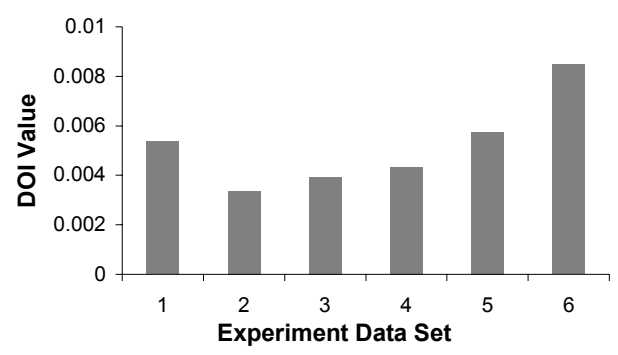

Figure 8: DOI Values from MICA2 Experiments

\subsubsection{Non-isotropic Properties in RIM Model}

Many models are used to estimate path loss, such as the free-space propagation model, the two-ray model and the Hata model [22]. These models are isotropic in the sense that the path losses in different directions are the same. To reflect the two main properties of radio irregularity, namely non-isotropic and continuous variation, we adjust the value of path loss models in Equation 1 based on DOI values, resulting in the following formula:

Received Signal Strength $=$ Sending Power - DOI Adjusted Path Loss + Fading

DOI Adjusted Path Loss $=$ Path Loss $\times K_{i}$

where $K_{i}$ is a coefficient to represent the difference in path loss in different directions. The coefficient $K_{i}$ is calculated in the following way:

$$
\begin{aligned}
& K_{i}=\left\{\begin{array}{l}
1, \quad i=0 \\
K_{i-1} \pm \text { Rand } \times \text { DOI }, 0<i<360 \wedge i \in N
\end{array}\right. \\
& \text { where }\left|K_{0}-K_{359}\right| \leq D O I
\end{aligned}
$$

We can generate $360 K_{i}$ values for the 360 different directions, based on Equation 4, by randomly fixing a direction as the 
starting direction represented by $i=0$. For the direction which doesn't have an integer value of angle from the start direction, we interpolate the $K_{i}$ value based on the values of the two adjacent directions which have integer angles from the starting direction.

$K_{i}=K_{s}+(i-s) \times\left(K_{t}-K_{s}\right)$

where $s=\lfloor i\rfloor \wedge t=\lceil i\rceil \bmod 360 \wedge 0<i<360 \wedge i \notin N$

The statistical analysis of our experimental data indicates that the variance of received signal strength in different directions fits the Weibull [7] distribution. The Weibull distribution can be used to model natural phenomena such as variation of wind speed, scattering of radiation, etc. The Rayleigh distribution, which is commonly used for modeling multi-path fading in wireless communication, is a special case of the Weibull distribution. Analysis details are provided in Appendix A. In Equation 4, we generate a random number according to the Weibull distribution.

\subsubsection{Heterogeneity Property in RIM Model}

Due to the difference in hardware calibration and battery status, received signal strength can be different from two sending nodes of the same type in the same experimental setting. In RIM, we use the variance of signal sending power to account for such a difference. We introduce a second parameter named VSP (Variance of Sending Power), which is defined as the maximum percentage variance of the signal sending power among different devices. The new signal sending power is modeled by the following equation:

VSP Adjusted Sending Power $=$ Sending Power $\times(1+$ Rand $\times$ VSP)

In Equation 6, we assume that the variance of sending power follows a Normal distribution, which is broadly used to measure the variance caused by the hardware.

With the two parameters: DOI and VSP, the RIM model can be formulated as follows:

Received Signal Strength $=$ VSP Adjusted Sending Power - DOI Adjusted Path Loss + Fading

With the help of the RIM model, we explore the impact of radio irregularity on MAC and routing protocols in the next two sections, respectively.

\section{IMPACT ON MAC LAYER}

In this section, we first analyze how operations in the MAC layer are affected by radio irregularity. We then quantify the degree of MAC performance degradation in the presence of radio irregularity.

\subsection{Logical Analysis of the Impact}

Most contention-based MAC protocols are based on carrier sensing or handshaking techniques. In this section, we analyze the impact of radio irregularity from the technical point of view.

- Impact on Carrier Sensing: Radio irregularity increases the chance for MAC protocols that use the carrier sensing technique to get involved in the hidden terminal problem. For example, in Figure 9 (a), while node B is transmitting packets to node $\mathrm{C}$, due to the irregularity, node $\mathrm{A}$ can not detect the signal from node B, so node A senses a clear channel and starts to transmit packets. As a result, a collision happens at receiver C. This scenario doesn't occur in isotropic radio situations, where node A detects node B's signal and refrains from transmitting the packets. Typical protocols using the carrier sensing technique are CSMA [18], MACA [15], MACAW [1] and 802.11 DCF [8].

- Impact on handshaking: The handshaking technique is specially designed to resolve hidden and exposed terminal problems. However, they can not resolve the hidden and exposed terminal problems due to asymmetry, which can be produced by radio irregularity. This can be demonstrated in an example (Figure 9 (b)). We assume that node A sends a RTS message to node $\mathrm{B}$, and then node $\mathrm{B}$ responds with a CTS message to node A. Any node overhearing the CTS message is supposed to wait long enough for node A to send out the data packet. If node C can't hear the CTS message from node $\mathrm{B}$ while node $\mathrm{B}$ can hear node $\mathrm{C}$, there will be a collision if node $\mathrm{C}$ sends data. Similar examples can be found for the exposed terminal case.

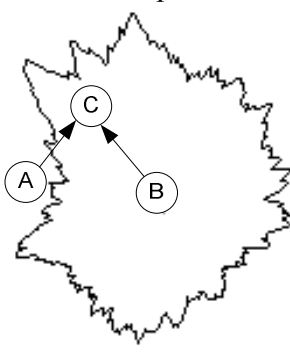

(a) Carrier Sensing

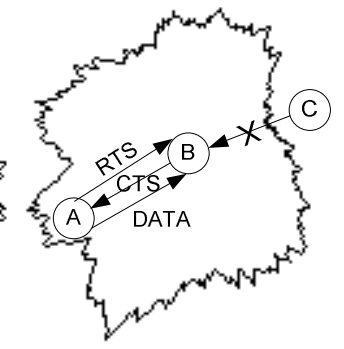

(b) Handshaking
Figure 9: Impact on MAC Protocols

\subsection{Quantitative Analysis of the Impact}

We implemented the RIM model in the radio layer of GloMoSim [27], a scalable discrete-event simulator developed by UCLA. We first describe our simulation configuration, and then evaluate the performance impact under different DOI and different VSP values, respectively.

\subsubsection{Simulation Configuration}

Table 1: Simulation Configuration

\begin{tabular}{|l|l|}
\hline TERRAIN & $(150 \mathrm{~m}, 150 \mathrm{~m})$ \\
\hline Node Number & 100 \\
\hline Node Placement & Uniform \\
\hline Payload Size & 32 Bytes \\
\hline Routing Protocol & AODV, DSR, GF \\
\hline MAC Protocol & CSMA, 802.11 (DCF) \\
\hline Radio Model & RIM \\
\hline Radio Bandwidth & $200 \mathrm{~Kb} / \mathrm{s}$ \\
\hline
\end{tabular}

In the experiments, we use six CBR streams as the workload and set the CBR rate at a low rate, in order to isolate the effect of congestion and radio irregularity. We choose two typical MAC protocols: CSMA and 802.11 DCF. Two metrics are used: 1) the loss ratio (number of frames lost / number of frames sent) and 2) the average single hop delay of received packets. We vary the 
DOI and VSP values ${ }^{2}$ separately in order to isolate and identify the impact individually. Each result shown in the graphs is an average of 140 runs. The $95 \%$ confidence intervals are within $0 \sim 25 \%$ of the mean.

In order to make our evaluation close to existing hardware proposed for use in wireless sensor network environments [24], we use the simulation configuration shown in Table 1 . In all experiments, we investigate the range of DOI values according to the experimental data obtained from MICA2 motes as shown in Figure 8 .

\subsubsection{MAC Performance with Different DOI}

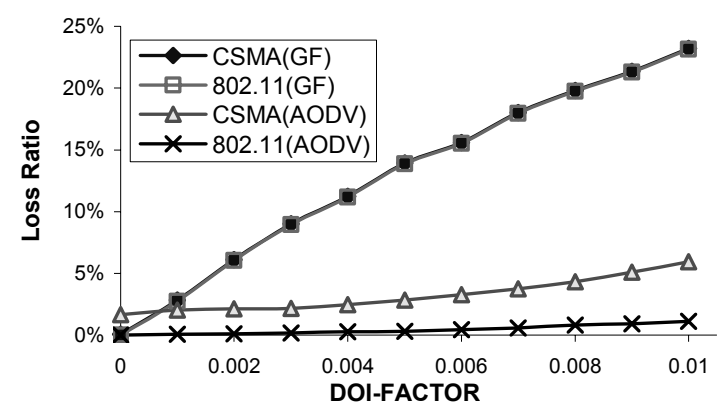

(a)Loss Ratio vs. DOI

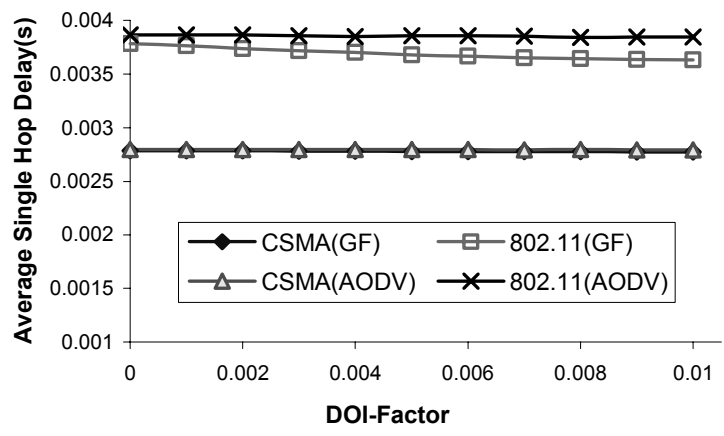

(b)Average Single Hop Delay vs. DOI

Figure 10: MAC Performances with Different DOI Values

In the initial setup, we use Geographic Forwarding (GF) for the routing layer and compare the MAC performance between 802.11 and CSMA. We found that the MAC loss ratio increases rapidly with the increase in DOI values (Figure 10 (a)). However, 802.11 and CSMA yield roughly the same results. We realize that MAC performance can be strongly affected by routing, because an incorrect routing decision might lead to the failure at MAC layer. For instance, the routing layer designates that the MAC layer send a packet to a node that is out of reach. So we repeat the experiments with the AODV protocol as the routing layer. We find that MAC loss ratio increases slightly with the increase of DOI. Such a discrepancy is a strong indication that the radio irregularity has a much larger impact on routing protocols than MAC protocols. We explain this in more detail in Section 6.

\footnotetext{
${ }^{2}$ Spherical radio model is configured by setting DOI to zero.
}

From Figure 10 (b), we can see that with the increase of DOI values, the average single hop delay remains almost the same. The reason is that increasing the DOI value only increases the communication asymmetry, but not the congestion. This is also a confirmation that packet loss in Figure 10 (a) is not due to congestion.

\subsubsection{MAC Performance with Different VSP}

In this experiment, we set the DOI value to zero, which means that the radio range is isotropic. However, different VSP values make radio ranges different among nodes.

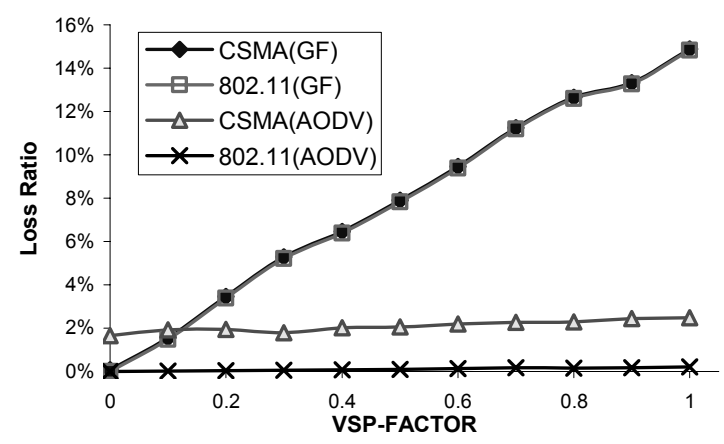

(a) Loss Ratio vs. VSP

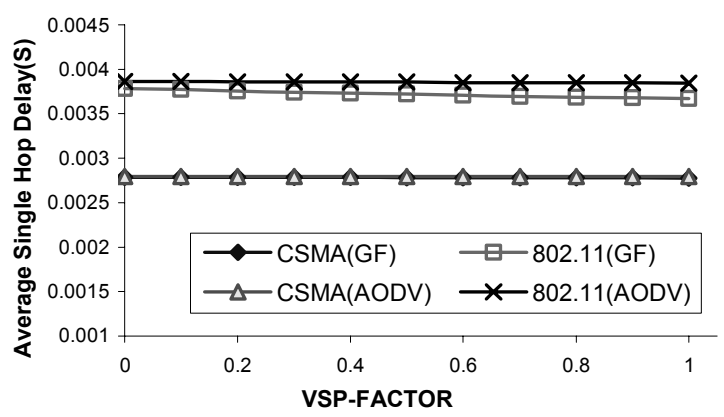

(b) Average Single Hop Delay vs. VSP

Figure 11: MAC Performances with Different VSP Values

The results shown in Figure 11 are similar to the results shown in Figure 10, which we obtain by varying the DOI values. The average single hop delay remains almost the same, because the different sending power only increases the degree of communication asymmetry, but does not increase the congestion.

The loss ratio increases with the increase of VSP values because the irregularity results in more asymmetric links. The loss ratio when AODV is used is much lower than when GF is used because asymmetric links have a larger impact on GF than on AODV. This result indicates that varying the VSP values has a much larger impact on routing protocols than on MAC protocols, which is similar to the behavior we observed by varying the DOI values.

\section{IMPACT ON ROUTING LAYER}

In this section, we analyze and quantify the impact of radio irregularity on routing protocols. We first discuss three techniques that are widely used in most routing protocols: path-reversal, 
multi-round discovery, and neighbor discovery. Our analysis shows that both path-reversal and neighbor-discovery are greatly influenced by radio irregularity. However, the multi-round discovery technique is able to deal with radio irregularity, but with relatively high overhead. Our simulation results also show that radio irregularity has a great impact on Geographic Forwarding (GF), but a small impact on AODV and DSR.

\subsection{Logical Analysis of the Impact}

We analyze the influence of radio irregularity on path-reversal, multi-round discovery, and neighbor-discovery techniques in this section.

\subsubsection{Impact on Path-Reversal Technique}

Protocols that use path-reversal technique are built based on the assumption that if there is a path from node A to node B, there is also a reverse path from node $B$ to node $A$. The path may consist of a single link or multiple links. Most on-demand routing protocols used in ad hoc networks such as AODV [21], DSR [14], Direct Diffusion [13] and LAR [19] depend on this technique.

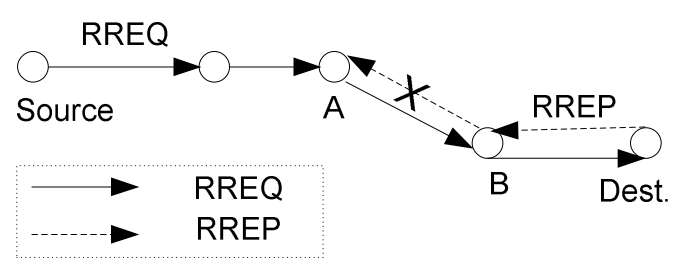

Figure 12: Impact on Path-Reversal Technique

Radio irregularity may result in asymmetric links and hence, it may have an adverse impact on protocols that use path-reversal techniques. For example, in Figure 12, node B can hear node A, but node A can not hear node B. So even though there is a path from source $\mathrm{S}$ to destination $\mathrm{D}$, we cannot assume that the reverse path from $D$ to $S$ exists. So during route discovery, if source $S$ broadcasts a route request (RREQ) to discover the path to destination $\mathrm{D}$, it may not be possible to deliver the reply (RREP) message to source $\mathrm{S}$ along the reverse path, even though node $\mathrm{D}$ replies to the request. In such a case, the route discovery fails.

The above analysis leads one to believe that it would be inappropriate to use any routing protocol that uses path-reversal in route discovery, such as AODV, DSR, DD and LAR, in an asymmetric environment, because they would have a very high loss ratio. However, the simulation results we present later show that AODV and DSR work reasonably well despite the asymmetric nature of communication. The reason is that in addition to path-reversal technique, these routing protocols also use the multi-round discovery, which is capable of dealing with asymmetry, but with a high overhead.

\subsubsection{Multi-Round Discovery Technique}

In AODV and DSR, the RREQ is broadcast towards the destination D. So node D receives RREQ messages from multiple paths, as shown in Figure 13. It chooses one of the many available paths to send the RREP message back to source S, according to some runtime configurable parameter, such as the RREQ arrival time, path load, or end-to-end delay of the path. If the reverse path doesn't exist, the RREP fails to arrive at sender S and the route discovery is repeated due to timeout. In the next attempt, thanks to the random nature of flooding, node $\mathrm{D}$ might receive a RREQ message from another path, which happens to be a symmetric connection.

The chance to establish a symmetric connection increases after retries. If there is no limitation on the number of retries, a symmetric path will sooner or later be discovered on the condition that such a path exists. We note that the rediscover technique provides a viable way to work around the effects of asymmetry, but with significant overhead.

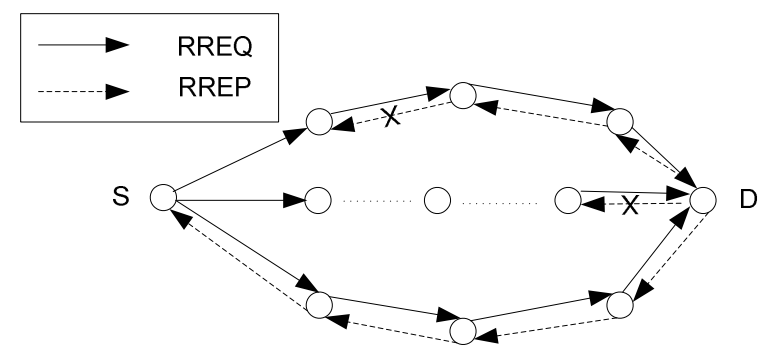

Figure 13: Route Discovery Using Rediscovery Technique

\subsubsection{Impact on Neighbor Discovery Technique}

Many location-based routing protocols [12] [16] [17] use the neighbor discovery technique in order to maintain the neighborhood information. However, the neighbor discovery technique works well only if the links are symmetric. For example, in Figure 14, node A discovers its neighbors by receiving beacons. Node A might choose one of its neighbors, node $\mathrm{B}, \mathrm{C}$, or $\mathrm{D}$ for forwarding packets. However, if node A picks node $B$ which is unable to hear node $A$, node $B$ will never receive the packet forwarded by node $\mathrm{A}$. If node $\mathrm{A}$ does not retry its transmission with the other neighbors, the transmission of the packet will fail. So the routing protocol based on the neighbor discovery technique is subject to failures when communication is asymmetric.

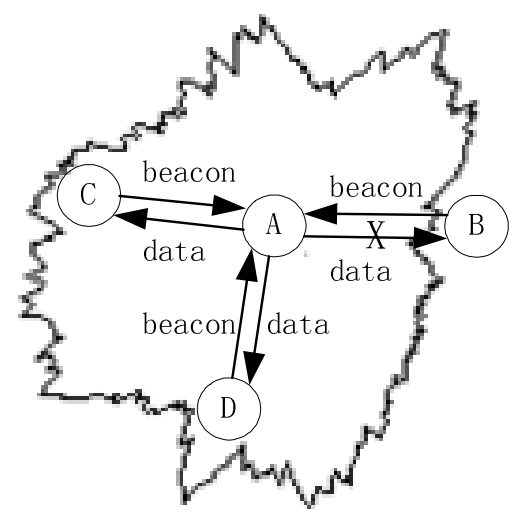

Figure 14: Impact on Neighbor Table Technique 


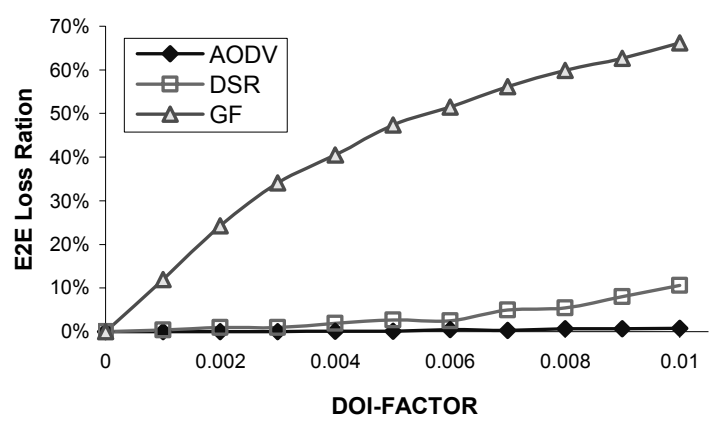

(a) E2E Loss Ratio vs. DOI

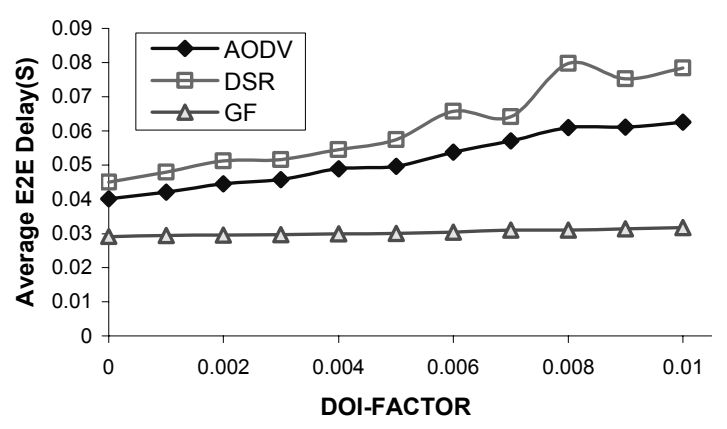

(b)Average E2E Delay vs. DOI

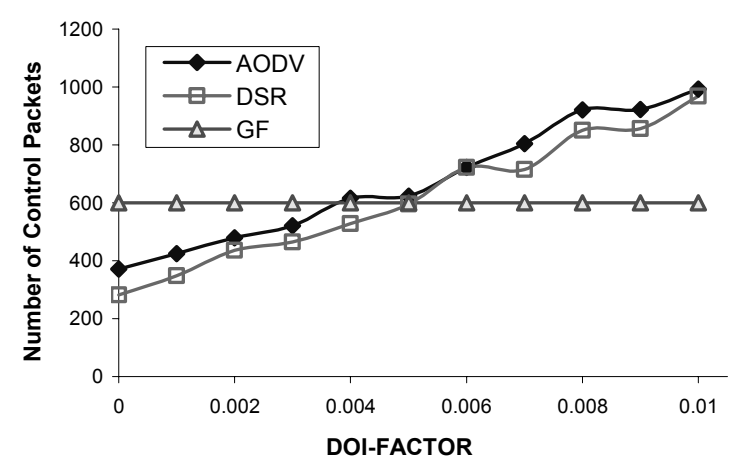

(c) Number of Control Packets vs. DOI

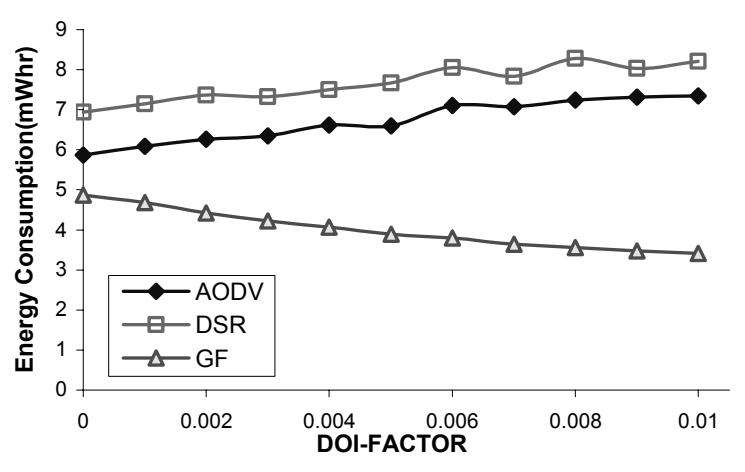

(d) Energy Consumption vs. DOI

Figure 15: Routing Performance with Different DOI

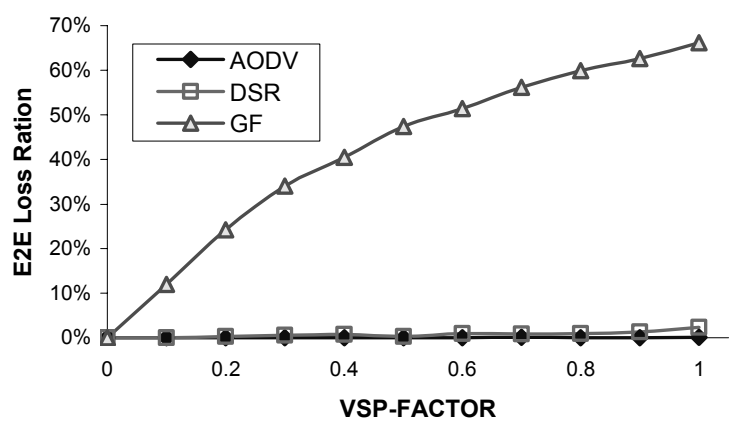

(a) E2E Loss Ratio vs. VSP

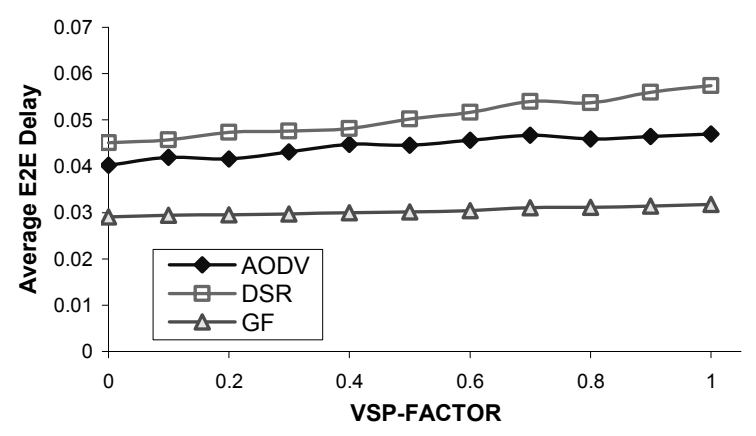

(b) Average E2E Delay vs. VSP

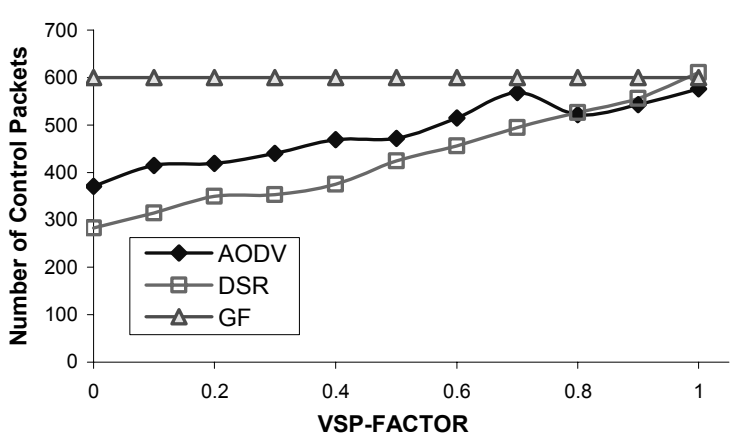

(c) Number of Control Packets vs. VSP

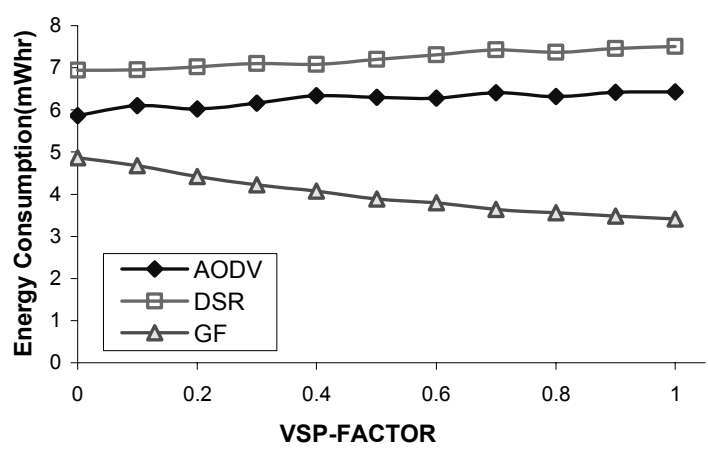

(d) Energy Consumption vs. VSP

Figure 16: Routing Performance with Different VSP 


\subsection{Quantitative Analysis of the Impact}

In this section, we quantify the performance penalty of radio irregularity. We conducted two sets of experiments by varying DOI and VSP. In each set, we measure four metrics: end-to-end (E2E) loss ratio, average E2E delay, number of control packets, and energy consumption.

\subsubsection{Routing Performance with Different DOI}

Figure 15 (a) shows that GF is greatly influenced by radio irregularity. It loses 70 percent of packets when the DOI is 0.01 . The reason is that according to the greedy forwarding rule, GF tends to choose a node near the border, which is more likely to have an asymmetric link with the sender. AODV and DSR perform well because they use multi-round discovery, exploring alterative paths to find a symmetric connection. However, they achieve a low loss ratio at the cost of increasing overhead in control packets shown in Figure 15 (c).

In Figure 15 (b), the average E2E delay of DSR and AODV increases with the increase in the DOI value. That is because more rounds of route discovery are needed as the radio irregularity increases. In Figure 15 (b) DSR has a higher delay than AODV, because the source routing technique in DSR adds the whole path in the header of data packets, which increases the transmission time. However, the E2E delay of GF remains the same because packets in GF either go through successfully or get lost.

Figure 15 (c) shows that while AODV and DSR need more control packets to do multi-round discovery, when the DOI value increases GF needs only a constant number of control packets for neighbor exchange.

From Figure 15 (d), we see that AODV and DSR consume more energy when DOI increases, because more control packets are used for rediscovery. However, GF transmits fewer data packets when the DOI value increases (Figure 15 (a)). Hence, the energy consumption reduces.

\subsubsection{Routing Performance with Different VSP}

In Figure 15, the impact of radio irregularity on the routing layer is measured for different DOI values. In this section, we measure the impact of radio irregularity on the routing layer by varying the VSP values. From our results, we find that an increasing value of VSP has a similar impact on AODV, DSR and GF, as an increasing value of DOI, because both lead to a higher degree of irregularity and therefore, a higher degree of link asymmetry.

From Figure 16 (a), we see that all routing protocols have higher loss ratio when the VSP value is increased, because there are more asymmetric links. GF has much higher loss ratio than AODV and DSR, because GF uses neighbor discovery and tends to choose the same node near the border of the radio range as the candidate, while AODV and DSR use multi-round discovery to try different paths.

As in the case of larger DOI values, larger VSP values result in more asymmetric links, which lead to larger average E2E delays (Figure 16 (b)) and higher energy consumption (Figure 16 (d)). However, GF does not require more beacons, so there is no increase in the control packets (Figure 16 (c)) and the delay remains the same (Figure 16 (a)). The energy consumption of GF reduces because GF drops more packets in the presence of asymmetry.
To summarize, as DOI and VSP increase, radio irregularity has a greater adverse impact on the GF protocol compared to ondemand routing protocols that use multi-round discovery such as AODV and DSR.

\section{SOLUTIONS FOR RADIO IRREGULARITY}

Having analyzed the causes and impact of radio irregularity, the key results can be summarized as follows:

- Radio irregularity is a common and non-negligible phenomenon in wireless communication.

- Radio irregularity has a greater impact on the routing layer than on the MAC layer.

- $\quad$ Routing protocols, such as AODV and DSR, that use multiround discovery technique, can deal with radio irregularity, but with a high overhead.

- $\quad$ Routing protocols, such as geographic forwarding, which are based on neighbor discovery technique, are severely affected by radio irregularity.

Based on both analytical and experimental results, we propose 6 potential solutions to improve the protocol performance in the presence of radio irregularity. We first describe the Symmetric Geographic Forwarding solution and the Bounded Distance Forwarding solution in detail and discuss their performance evaluation. We then follow that by briefly describing four other solutions.

\subsection{Symmetric Geographic Forwarding}

In location-based protocols, such as GF and GPSR, the beacon message only contains the node's ID and position. In the Symmetric Geographic Forwarding (SGF) solution, we allow a node to add the IDs of all its neighbors it has discovered into the beacon message. When a node receives a beacon message, it registers the sender as its neighbor in its local neighbor table, and then checks whether its own ID is in the beacon message. If the receiver finds its own ID in the neighbor list in the beacon message, then it marks the communication link connecting it to the sender as "SYMMETRIC". Otherwise, it marks the communication link between them as "ASYMMETRIC". Whenever a node needs to forward a packet, it selects only those neighboring nodes with which it is connected through "SYMMETRIC" links. Here we must emphasize that when a node broadcasts a beacon message, it should add the IDs of the nodes with which it has "SYMMETRIC" connectivity as well as those nodes with which it has "ASYMMETRIC" connectivity.

The SGF provides a basic prototype of incorporating symmetric detection into routing protocols. More sophisticated algorithms, such as measuring link quality with multiple rounds, can be introduced to deal with engineering issues in running systems.

We simulate SGF in GloMoSim. We find that SGF maintains most of the advantages of GF, such as scalability, and the absence of flooding. Furthermore, SGF is able to deal with asymmetry as effectively as the multi-path route discovery protocols, such as AODV and DSR, but at lower cost. The simulation setup use the same configuration as mentioned in Table 1. 


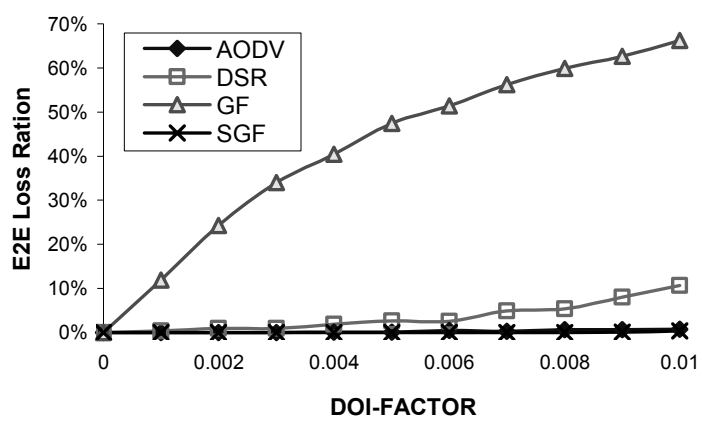

(a) E2E Loss Ratio vs. DOI

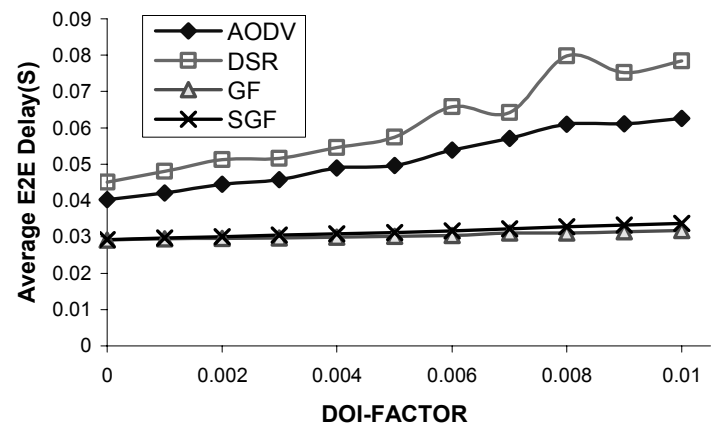

(b)Average E2E Delay vs. DOI

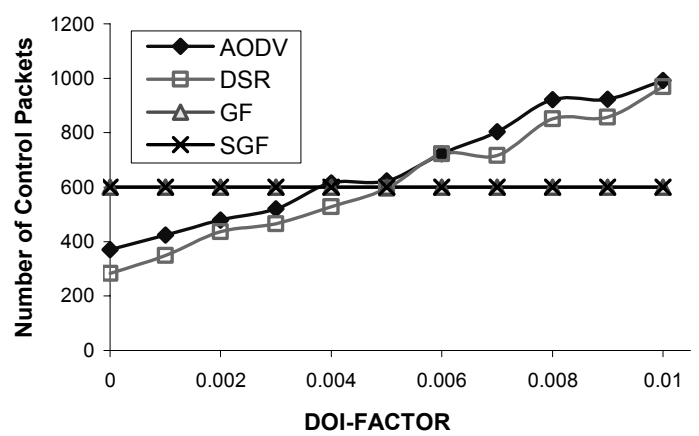

(c) Number of Control Packets vs. DOI

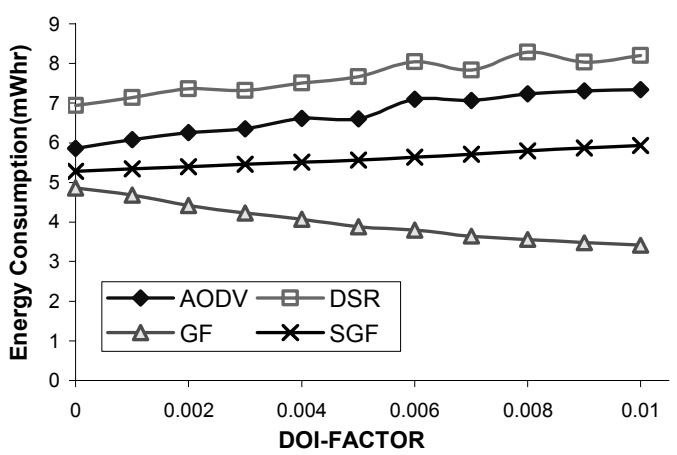

(d) Energy Consumption vs. DOI

Figure 17: SGF Performance with Different DOI

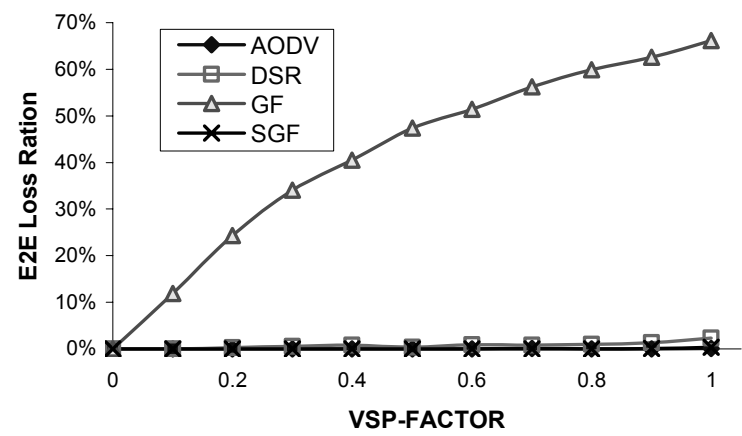

(a) E2E Loss Ratio vs. VSP

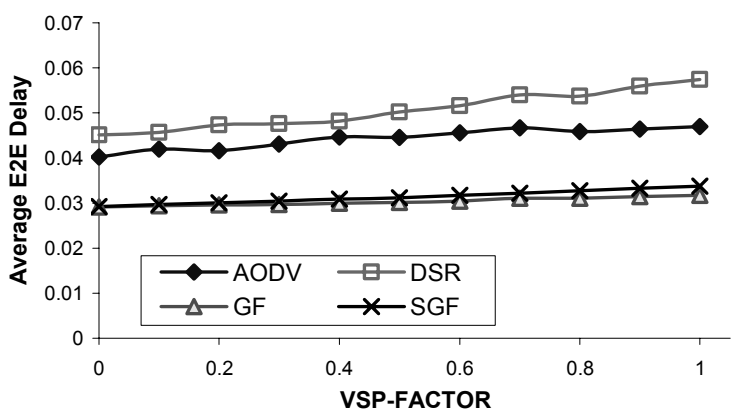

(b) Average E2E Delay vs. VSP

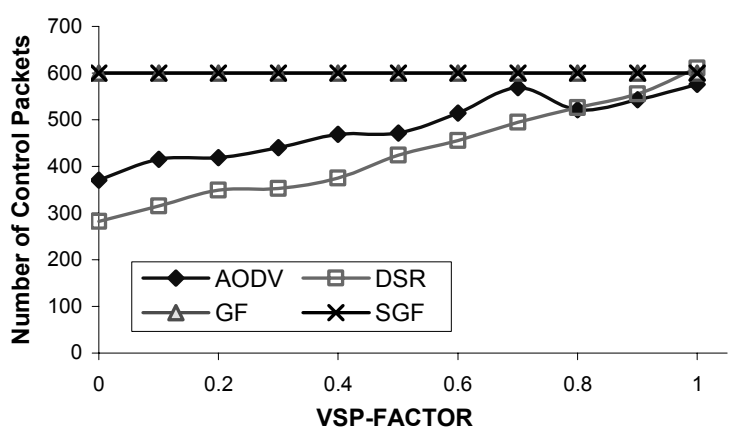

(c) Number of Control Packets vs. VSP

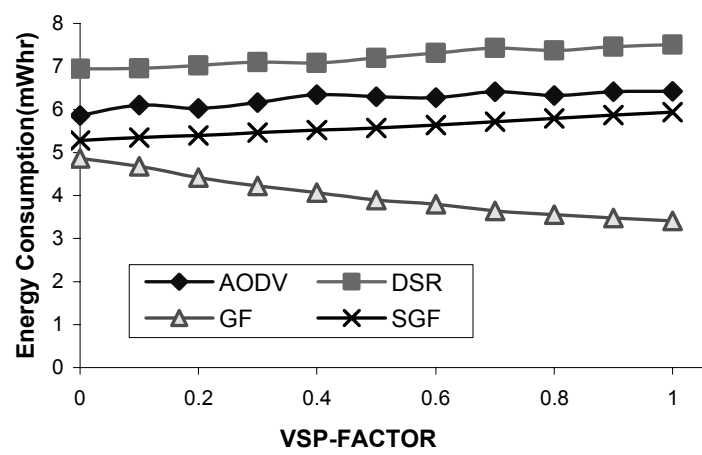

(d) Energy Consumption vs. VSP

Figure 18: SGF Performance with Different VSP 


\subsubsection{SGF Performance with Different DOI}

In this experiment, we incrementally increase the degree of irregularity (DOI) to measure the SGF performance.

From Figure 17 (a), we observe that SGF has significantly lower loss ratio than GF, and performs as well as AODV. This is because it avoids forwarding data along asymmetric links. From Figure 17 (b), we observe that SGF has almost the same average E2E delay as GF. The delay is much lower than that of ADOV and DSR. An interesting point from Figure 17 (c) is that SGF consumes the same number of control packets as GF, and the number of control packets remains the same with the increase of DOI value. From Figure 17 (d), it is clear that SGF consumes almost the same amount of energy with the increase of DOI. The reason is that as DOI increases, AODV and DSR require more control packets, while SGF maintains the same number of control packets. As a result, the energy consumption of AODV and DSR increases with DOI, while that of SGF almost remains the same. The energy consumption of SGF is larger than that of GF on account of two reasons. First, SGF needs a larger packet to piggyback the neighbors' IDs, even though both GF and SGF transmit the same number of control packets. Second, unlike GF, SGF does not drop packets with the increasing DOI. Hence, while the energy consumption of GF decreases, because GF drops useful data packets, the energy consumption of SGF almost remains the same.

\subsubsection{SGF Performance with Different VSP}

Similar conclusions can be drawn from the results in Figure 18. Compared with GF, SGF has much lower loss ratio, almost the same average E2E delay, and the same number of control packets, with an increase of the VSP value. The loss ratio of SGF is comparable to that of AODV and DSR. However, SGF has a much lower average E2E delay, constant number of control packets, and lower energy consumption. SGF consumes almost constant energy with an increase of the VSP value. In contrast, GF consumes less energy because it drops data packets, while AODV and DSR consume more energy because of a higher number of control packets.

To summarize, the SGF protocol not only maintains GF's scalability, but also successfully deals with radio irregularity. Compared with AODV and DSR, it achieves similar delivery ratio in the presence of radio irregularity with a lower E2E delay, a lower number of control packets and lower energy consumption.

\subsection{Bounded Distance Forwarding}

Bounded Distance Forwarding restricts the distance over which a node can forward a message in a single hop. It can act as an addon rule to many routing protocols. The distance bound is decided based on the degree of radio irregularity of the real devices in a physical system.

We add the Bounded Distance Forwarding rule on the spanning tree module in a vehicle tracking system [11] in which we deploy 60 MICA2 motes. In the experiments, we incrementally increase the single hop forwarding bound from 8 feet to 100 feet and count the number of nodes that report their status and Figure 19 shows this data as a percentage of the total number of nodes deployed. Data points here are average values over five runs. Figure 19 indicates two interesting phenomena. First, when we use a very low forwarding bound ( 8 feet) to eliminate the asymmetric links, the performance, however, is not good. This is because relative node density decreases when the enforced communication range is small. Hence, the chance of a network partition increases. Moreover, a smaller forward bound per hop leads to a longer route, thus a higher chance of loss. Second, when the forwarding bound reaches larger values (16 100 feet), link asymmetry becomes the dominating factor. Figure 19 shows that when the forwarding bound is 16 feet, we receive almost every report. This bound is about half of the MICA2 radio range on the ground. Above 16 feet, performance reduces monotonically because of increase in link asymmetry.

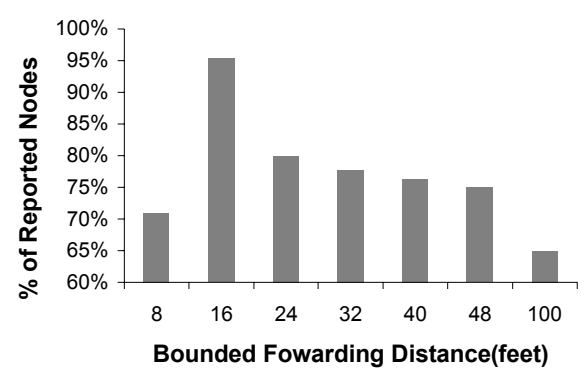

Figure 19: Percentage of Reporting Nodes

\subsection{Other Solutions}

In this section, we propose four additional potential solutions to deal with radio irregularity.

- Bidirectional Flooding: The multi-round discovery technique can deal with radio irregularity. However, it needs multiple rounds of flooding to explore different paths, which can be very expensive. In Bidirectional Flooding, the source propagates the RREQ towards the destination through flooding. After the destination receives the RREQ, it propagates the RREP to the source through flooding, instead of using the reverse path along which it received the RREQ from the source. Multi-round discovery can not guarantee finding symmetry connections within a bounded number of flooding stages. In contrast, bidirectional flooding completes the discovery by flooding twice.

- Learning Function: In an earlier section we mentioned that GF has a higher loss ratio than AODV and DSR, because GF tends to choose the same candidate near the border to forward packets to a destination, while AODV and DSR attempt different paths due to the nature of flooding. To address this shortcoming of GF, we can enhance GF with a learning function, which allows a node to make better decisions based on previous routing failures. In the learning function, we distinguish the routing failures arising due to congestion from those that arise due to asymmetric links. This can be done with the help of the 802.11 (DCF) in the MAC layer. If a node receives the CTS, but not the ACK, then the link should be symmetric and the routing failure might be a result of congestion. Such a failure can be solved by retransmissions. However, if a node fails to receive the CTS despite several retransmissions, then the chances are that the link is asymmetric. This learning function allows a node to remember such an asymmetric link and to avoid trying it again. 
- RTS Broadcast: Another solution we propose is called the RTS Broadcast, which involves both the MAC and routing layers. We first broadcast a special RTS message, which sets the destination as ANY_NODE. Any node hearing it backs off for a random amount of time and replies with a CTS message. Among all the nodes that send the CTS message, the one that is closest to the destination is chosen as the forwarding candidate. Since the RTS and CTS detect connectivity along the forward and reverse directions of a channel, forwarding packets along asymmetric channels can be avoided.

- High Energy Asymmetry Detection: IEEE 802.11 (DCF) uses a collision-avoidance strategy in which any node upon hearing an RTS, CTS, or DATA message defers its transmission until the data is sent out. However, a node can still interfere with the message transmission even though it is not able to hear any of the RTS, CTS and DATA messages in the presence of asymmetry. The sixth solution we propose is to send out a High Energy Asymmetry Detection (HEAD) control message which has a higher sending power than the other control messages. So more nodes will hear the highpowered signal, and prevent themselves from sending messages. The HEAD message is sent out before the RTS message. Any node other than the destination, upon hearing the HEAD message, sets its NAV to a value large enough so that data can be sent out without contention. The wait time and destination ID are included in the HEAD message. Conflicts may arise if two nodes send out the HEAD messages simultaneously. That is resolved in a manner similar to the way to resolve conflicts arising from the simultaneous transmission of two RTS messages. Hence, the transmission sequence is modified from RTS-CTS-DATAACK to HEAD-RTS-CTS-DATA-ACK. While the higher sending power of the HEAD message lowers the collision rate, it also reduces the channel utilization. This tradeoff between collision rate and desired channel utilization can be balanced by choosing an appropriate value for the sending power.

We note that the solutions we mentioned above are still open topics and require further refinements. Extensive analysis and evaluation in the future are required to demonstrate their applicability and effectiveness.

\section{CONCLUSIONS AND FUTURE WORK}

In this paper, we confirm the existence of radio irregularity which is the main focus of several recent research papers [4][9][23][28]. Our contributions are as follows:

1. To the best of our knowledge, our work is the first to bridge the gap between isotropic radio models assumed by most simulators and the non-isotropic radio properties in reality.

2. We propose a novel RIM model that approximates three essential properties exhibited in radio irregularity: nonisotropy, continuous variation and difference in sending power.

3. We implement the RIM model in GloMoSim, and run a set of simulation experiments to investigate radio irregularity's impact on MAC and routing layer performance. We discover that, among the protocols we evaluate, the radio irregularity has a greater impact on the routing layer than MAC layer.
We also discover that radio irregularity has a greater impact on location-based routing protocols than on-demand protocols that use multi-round discovery technique.

4. Finally, we propose six potential solutions. We implement SGF in GloMoSim, and implement the Bounded Distance Forwarding rule in a real running tracking system consisting of 60 MICA2 motes. From the data we collect from the simulator and the running system, we find that SGF and Bounded Distance Forwarding can successfully deal with radio irregularity.

In future work, we will concentrate on the following aspects. First, we plan to further refine the RIM model and incorporate our work into the standard release of GloMoSim and NS-2. Second, we plan to analyze the impact of radio irregularity on other protocols, such as localization and topology control. Third, we plan to analyze and evaluate the remaining four approaches mentioned in Section 7.3.

\section{Acknowledgements}

The authors would like to thank the MobiSys shepherd, Hari Balakrishnan, and the anonymous reviewers for their helpful suggestions that improved our work. This research was supported by the DARPA IXO offices under the NEST project (grant number F336615-01-C-1905).

\section{REFERENCES}

[1] V. Bharghavan, A. Demers, S. Shenker and L. Zhang, MACAW: A Media Access Protocol for Wireless LANs, In Proc. of ACM SIGCOMM, pp. 212-225, 1994.

[2] N. Bulusu, J. Heidemann and D. Estrin, GPS-less Low Cost Outdoor Localization for Very Small Devices, In IEEE Personal Communications Magazine, pp. 28-34, October 2000.

[3] A. Cerpa and D. Estrin, ASCENT: Adaptive SelfConfiguring Sensor Networks Topologies, In Proc. of the IEEE Infocom, 2002.

[4] A. Cerpa, N. Busek and D. Estrin, SCALE: A Tool for Simple Connectivity Assessment in Lossy Environments, In CENS Technical Report 0021, September 2003.

[5] B. Chen, K. Jamieson, H. Balakrishnan and R. Morris, Span: An Energy-Efficient Coordination Algorithm for Topology Maintenance in Ad-hoc Wireless Networks, In $A C M$ MobiCom, July 2001.

[6] Chipcon CC1000 Low Power Radio Transceiver, http:// www.chipcon.com/files/cc1000_data_sheet_2_1.pdf

[7] J. L. Devore, Probability and Statistics for Engineering and the Sciences, Brooks/Cole Publishing, 1982.

[8] IEEE 802.11, Part II: Wireless LAN Medium Access Control (MAC) and Physical Layer (PHY) Specification, ANSI/IEEE Std. 802.11, 1999.

[9] D. Ganesan, B. Krishnamachari, A. Woo, D. Culler, D. Estrin and S. Wicker, Complex Behavior at Scale: An Experimental Study of Low-Power Wireless Sensor Networks, In Technical Report UCLA/CSD-TR 02-0013, 2002. 
[10] T. He, C. Huang, B. M. Blum, J. A. Stankovic and T. F. Abdelzaher, Range-Free Localization Schemes in Large Scale Sensor Networks, In Proc. MOBICOM, 2003.

[11] T. He, S. Krishnamurthy, J. A. Stankovic, T. F. Abdelzaher, L. Luo, R. Stoleru, T. Yan, L. Gu, J. Hui, B. Krogh, EnergyEfficient Surveillance System Using Wireless Sensor Networks, In ACM MobiSys 2004, Boston, MA, June 2004.

[12] T. He, J. A. Stankovic, C. Lu and T. F. Abdelzaher, SPEED: A Stateless Protocol for Real-Time Communication in Sensor Networks, In IEEE ICDCS 2003, Providence, RI, May 2003.

[13] C. Intanagonwiwat, R. Govindan and D. Estrin, Directed Diffusion: A Scalable and Robust Communication Paradigm for Sensor Networks, In Proc. MOBICOM, pp. 56-67, March 2000 .

[14] D. B. Johnson and D. A. Maltz, Dynamic Source Routing in Ad Hoc Wireless Networks, Mobile Computing, edited by Tomas Imielinski and Hank Korth, Kluwer Academic Publishers, ISBN: 0792396979, 1996.

[15] P. Karn, MACA - A New Channel Access Method for Packet Radio, In ARRL/CRRL Amateur Radio 9th Computer Networking Conference, pp. 134-140, 1990.

[16] B. Karp and H. T. Kung, GPSR: Greedy Perimeter Stateless Routing for Wireless Networks, In Proc. MOBICOM, pp. 243-254, August 2000.

[17] B. Karp, Geographic Routing for Wireless Networks, Ph.D. Dissertation, Harvard University, Cambridge, MA, October 2000.

[18] L. Kleinrock and F. A. Tobagi, Packet Switching in Radio Channels: Part I - Carrier Sense Multiple-access Modes and Their Throughput-delay Characteristics, In IEEE Trans. Commun., vol. COM-23, pp. 1400-1416, Dec. 1975.

[19] Y. B. Ko and N. H. Vaidya, Location-Aided Routing (LAR) in Mobile Ad Hoc Networks, In Proc. MOBICOM, pp. 6675, 1998.

[20] D. Niculescu and B. Nath, DV Based Positioning in Ad hoc Networks, In Journal of Telecommunication Systems, 2003.

[21] C. E. Perkins, and E. M. Royer, Ad-hoc On-Demand Distance Vector Routing, In Proc. of the 2nd IEEE Workshop on Mobile Computing Systems and Applications, pp. 90-100, February 1999.

[22] P. M. Shankar, Introduction to Wireless Systems, John Wiley \& Sons, Inc, ISBN 0-471-32167-2, 2001.

[23] A. Woo, T. Tong and D. Culler, Taming the Underlying Challenges of Reliable Multihop Routing in Sensor Networks, In ACM SenSys 2003, Los Angeles, CA, November 2003.
[24] XBOW MICA2 Mote Specifications: http://www.xbow.com/

[25] Y. Xu, J. Heidemann and D. Estrin, Geography-informed Energy Conservation for Ad Hoc Routing, In $A C M$ MOBICOM 2001, Rome, Italy, July 2001

[26] T. Yan, T. He, J. A. Stankovic, Differentiated Surveillance for Sensor Networks, In ACM SenSys 2003, Los Angeles, CA, November 2003.

[27] X. Zeng, R. Bagrodia and M. Gerla, GloMoSim: a Library for Parallel Simulation of Large-scale Wireless Networks, In Proc. of the 12th Workshop on Parallel and Distributed Simulations, May 1998.

[28] Y. J. Zhao and R. Govindan, Understanding Packet Delivery Performance in Dense Wireless Sensor Network, In $A C M$ SenSys 2003, Los Angeles, CA, November 2003.

\section{Appendix A}

We use the goodness-of-fit statistical testing to determine the statistical distribution of the variance of the received signal strength (in $\mathrm{dBm}$ ) per degree in the direction that is obtained in our experiments. We find that among different continuous distributions, the Weibull distribution [3] has the maximum likelihood of matching our experimental data. A random variable $\mathrm{X}$ that has a Weibull distribution with parameters has a probability density function defined by the following equation, where $a$ is the shape parameter and $b$ is the scale parameter.

$f(x)= \begin{cases}\left(a / b^{a}\right) \times x^{(a-1)} \times e^{-\left(\frac{x}{b}\right)^{a}}, & x \geq 0 \\ 0, & x<0\end{cases}$

Table 2 shows the likelihood values and the parameters of the Weibull distribution that fits our experimental data. These values are computed at a $95 \%$ confidence level.

Table 2: Data fitting to the Weibull distribution

\begin{tabular}{|l|l|l|l|}
\hline & Likelihood & $\mathrm{a}$ & $\mathrm{b}$ \\
\hline Dataset 1 & 48.55 & 1.13 & 0.28 \\
\hline Dataset 2 & 154.43 & 1.01 & 0.17 \\
\hline Dataset 3 & 145.25 & 0.86 & 0.18 \\
\hline Dataset 4 & 277.44 & 0.67 & 0.16 \\
\hline Dataset 5 & 204.51 & 0.58 & 0.17 \\
\hline Dataset 6 & 111.15 & 0.53 & 0.22 \\
\hline
\end{tabular}

